\title{
CAN VOICE ONSET TIME EXPLAIN VOWEL DURATION VARIATION?
}

\author{
1Ziyad Rakan Kasim \\ ${ }^{1}$ Department of English, College of Education for Humanities, University of Mosul, Iraq
}

\begin{abstract}
Vowel durations have been reported in many languages to vary before syllable final stops; these durations are shorter before voiceless stops and longer before voiced ones. One type of explanations to this phenomenon suggests prevocalic effect influencing vowel duration variation. The present study attempts at a preliminary investigation to find out whether the voice onset time (VOT) of a syllable initial stop can be correlated with this vowel duration variation in a CVC context in Arabic. Measurements of the VOT of the Arabic voiceless uvular stop /q/ are made where /q/ is the first C, V is one of /a:/, / u:/ and /i:/ and the final $\mathrm{C}$ is either $/ \mathrm{t} /$ or $/ \mathrm{d} /$. A Paired-Samples $\mathrm{T}$-Test is used to compare between the VOT measurements of / $q$ / of the words containing / $t$ / and those containing / $d$ /. The result of the comparison did not show any significant difference; no relationship could be found between the VOT of the initial stop and vowel duration variation.
\end{abstract}

Keywords: Arabic, stops, vot, vowel duration.

\section{Introduction}

Vowel duration variation is a phenomenon that has been reported in many languages, where vowels tend to be longer before, especially, a syllable final voiced stop and shorter before a voiceless one (Ladefoged \& Maddieson, 1996; Reetz \& Jongman, 2009), among others. Many explanations have been put forward to account for this variation, (see Cho \& Ladefoged; 1999, Maddieson, 1997) for a survey. One of these explanations is closure duration of the following stop. It has been reported that closure duration may be correlated with vowel durational differences (Port \& Dalby, 1982).

Although most of the explanations offered to justify vowel duration variation focus on what follows the vowel (see, for example, Tauberer \& Evanini, 2009), a few studies have examined prevocalic effect (Naeser,

Academic Journal of Nawroz University

(AJNU) Volume 8, No 4 (2019).

Regular research paper : Published 20 Dec 2019

Corresponding author's e-mail : zyad.rakan@gmail.com

Copyright (C2018 Ziyad Rakan Kasim.

This is an open access article distributed under the Creative

Commons Attribution License.
1970; Van Santen, 1992; Allen \& Miller, 1999; Metz, et al. 2006). It has been shown that vowel durations are longer following a voiced consonant compared to a voiceless one (Naeser, 1970; Metz, et al. 2006). Moreover, Allen and Miller (1999) found that a change from voiced to voiceless stop resulted in an increase in VOT and a decrease in vowel duration.

Arabic is among the languages that shows vowel durational differences before final voiceless and voiced stops. In a previous study, the durations of the three Arabic long vowels /a:/, /u:/ and /i:/ have been investigated preceding /t/ and /d/ (Kasim, 2014a). The results show that the three vowels have longer durations before $/ \mathrm{d} /$ and shorter durations before $/ \mathrm{t} /$. In addition, closure duration is also investigated, in the same study, in an attempt to examine the relation between vowel durational differences and closure duration. However, no correlation is found. The three vowels are investigated in a CVC context where the initial consonant is the voiceless uvular stop /q/.

Since prevocalic voiced and voiceless consonants could 
have some effect on vowel duration, the focus of the present study will be on investigating this effect in Arabic. More specifically, emphasis will be on the voicelessness of a prevocalic stop, viz. /q/, concentrating on the VOT of the stop in a CVC context. The VOT of the initial stop will be investigated in CVC words ending with $/ t /$ and $/ d /$ in hope of finding a relationship that might be established between the VOT of the initial stop and the duration variation of the following vowel.

\section{Aim of the Study}

The aim of the study is to examine prevocalic effect of an initial stop on the following vowels in CVC words ending with voiceless and voiced stops. More specifically, the aim is to investigate if there is a relation between the VOT of a syllable initial voiceless stop consonant, i.e. /q/, and vowel durational differences found in syllables ending with voiceless and voiced stops, i.e. /t/ and /d/ in Arabic. In other words, can the VOT of the initial stop be linked to, and thus used to explain, the variation of vowel duration preceding voiceless and voiced consonants? The aim is achieved by comparing the VOT of /q/ found in words ending with / $\mathrm{t} /$ with those words ending with / $\mathrm{d} /$.

\section{Hypothesis}

It is hypothesized that the initial stop immediately preceding the vowel, i.e. /q/, has greater VOT values in words where the final consonant is voiceless, i.e. / $t$ / (thus compensating vowel shortening) and lower values in words where the final consonant is voiced, i.e. /d/ (thus compensating vowel lengthening). Thus, the null hypothesis is $(\mathrm{H} 0: \mu 1=\mu 2)$, where $\mu 1$ and $\mu 2$ represent /q/ VOT values in words containing final $/ t /$ and /d/, respectively; i.e. there is no significant difference between /q/ VOT values in words containing final $/ \mathrm{t} /$ and $/ \mathrm{d} /$. The alternative hypothesis is $(\mathrm{H} 1: \mu 1 \neq \mu 2)$, i.e. there is a significant difference between /q/ VOT values in words containing final $/ \mathrm{t} /$ and $/ \mathrm{d} /$. The level of significance chosen is (0.05).

\section{Method}

\subsection{Material}

Three Arabic CVC pairs of words are used to test the research hypothesis. The initial $\mathrm{C}$ is /q/, the $\mathrm{V}$ is one of /a:/, /u:/ and /i:/, and the final $\mathrm{C}$ is either / $\mathrm{t} /$ or $/ \mathrm{d} /$. Two of the pairs are minimal pairs (/qa:t/-/qa:d/, /qu:t/-/qu:d/), the other pair (/qi:t/-/qi:d/) contains nonsense words that are cut from the two meaningful words / maqi:t/-/faqi:d/; see (Table 1) for the test data. The reason why all the three Arabic long vowels are selected is to examine whether vowel quality has any relation with the VOT of the initial stop. It should be noted that the data used in the present study are the same data used by (Kasim, 2014a). The reason why the same data are utilized is for comparison consistency.

Table 1: The data used in the test.

\begin{tabular}{|l|l|l|l|}
\hline \multicolumn{1}{|c|}{ Token } & \multicolumn{1}{|c|}{ Gloss } & \multicolumn{1}{c|}{ Token } & \multicolumn{1}{c|}{ Gloss } \\
\hline /qa:t/ & plant (n.) & /qa:d/ & (he) lead (v. past) \\
\hline /qu:t/ & food & /qu:d/ & drive (v. imperative) \\
\hline /ma'qi:t/ & hateful & /fa'qi:d/ & deceased \\
\hline
\end{tabular}

\subsection{Subjects}

Ten native speakers of Iraqi (Mosuli) Arabic are selected for this study (three females and seven males). All of them are educated. Their ages range between 20 and 45 years. None of them has any speech disorders.

\subsection{Equipment}

In order to record the test data, a laptop PC is used with a desktop microphone (type Logitech). The recording software used is Praat which is also used for VOT measurements. The recording sampling rate is 44100 $\mathrm{Hz}$ using a mono channel. For the statistical analysis, the IBM SPSS Statistics 19 is used.

\subsection{Testing procedure}


The test words were printed in Arabic on flash cards (4 $\mathrm{cm} \times 11.5 \mathrm{~cm}$ ) using Times New Roman with a font size of 100 points. Each subject was presented with the test words and was asked to familiarize himself/herself with the words before recording. In addition, the researcher explained to the subjects the need to extract the second syllables from the last two words, i.e. /ma'qi:t/ and /fa'qi:d/ (see Table 1), and obtain the nonsense pair /qi:t/ and /qi:d/ (on separate flash cards) for comparison reasons. This last pair was added to the list of test words. Thus, the total number of flash cards used was eight. Each subject was asked to pronounce each test word in the carrier sentence / ' Pana smis' tu:ha / "I heard it (as) " when the researcher presented the flash card. The subjects were asked to pronounce the carrier sentence in natural everyday speech without making any pauses between words. The test words were then randomized and presented again to the subjects. The microphone was about $10 \mathrm{~cm}$ from the subject's mouth. There were three rounds of recording for each subject. Thus, the total number of tokens obtained was 240 (8 words times 3 trials times 10 subjects). However, the tokens of the near minimal pair /ma'qi:t/-/fa'qi:d/ were not included in the analysis of the test, so that the final number of tokens used in the present study was (180). Each subject's performance was recorded individually in a quiet room.

\subsection{Acoustic measurements}

It should be noted that measurements of the /q/ VOT values were not always possible. Two reasons made such measurements unattainable. First, in some cases the burst of the stop was not clear. Secondly, in other cases there were some intermittent striations of vocal fold vibrations within the burst of the stop. These conditions prevented measuring VOT of the initial stop. As such, there were missing VOT values in the test data. To compensate for these missing VOT values the average was used for statistical analysis. The VOT measurements of the initial /q/ are given in the Appendix.

\section{Result}

In order to find out whether a correlation exists between the VOT of the initial /q/ in words containing final voiceless and voiced stops, a Paired-Samples TTest is used. Table 2 below summarizes the data statistics.

Table 2: Paired-Samples statistics of the three pairs containing/q/.

\begin{tabular}{|c|c|c|c|c|c|}
\hline \multicolumn{2}{|c|}{} & Mean & N & $\begin{array}{c}\text { Std. } \\
\text { Deviation }\end{array}$ & Std. Error Mean \\
\hline \multirow{4}{*}{ Pair 1 } & qa:t & 28.1667 & 30 & 8.24238 & 1.50485 \\
\cline { 2 - 6 } & qa:d & 28.0667 & 30 & 9.69868 & 1.77073 \\
\hline \multirow{3}{*}{ Pair 2 } & qu:t & 29.0667 & 30 & 10.09586 & 1.84324 \\
\cline { 2 - 6 } & qu:d & 29.0667 & 30 & 9.78011 & 1.78560 \\
\hline \multirow{3}{*}{ Pair 3 } & qi:t & 27.7000 & 30 & 12.03486 & 2.19726 \\
\cline { 2 - 6 } & qi:d & 28.3667 & 30 & 10.89917 & 1.98991 \\
\hline
\end{tabular}

It is noticed from Table 2 that the VOT mean values for the first pair are almost the same, those of the second pair are identical, and there is very slight difference in the VOT means of the third pair of words. Table 3 below shows the Paired-Samples T-Test result.

Table 3: Summary of the comparison result of the /q/ VOT values in words containing/t/ and /d/.

\begin{tabular}{|l|l|c|c|c|}
\hline & & $\mathrm{T}$ & $\mathrm{Df}$ & Sig. (2-tailed) \\
\hline Pair 1 & qa:t - qa:d & .059 & 29 & .954 \\
\hline Pair 2 & qu:t - qu:d & .000 & 29 & 1.000 \\
\hline Pair 3 & qi:t - qi:d & -.307 & 29 & .761 \\
\hline
\end{tabular}

Table 3 shows that the $\mathrm{P}$ value is greater than 0.05 for all pairs $(\mathrm{P}>0.05)(.954,1.000, .761$ for /qa:t/-/qa:d/, /qu:t/-/qu:d/, and /qi:t/-/qi:d/, respectively). In this 
case the null hypothesis holds (see section 3); i.e. there is no significant difference of /q/ VOT values in words containing final $/ \mathrm{t} /$ and $/ \mathrm{d} /$.

\section{Discussion}

The investigation of the VOT of the initial stop /q/ did not show any significant influence on vowel duration variation in words containing final $/ \mathrm{t} /$ and $/ \mathrm{d} /$, as far as the data examined in this study are concerned. One reason why /q/ VOT values did not display any significant difference could be attributed to the shortness of these VOT values. In the test data, /q/ VOT mean values ranged between 27-29 ms. (see Table 2). In an experimental study on aspiration in Arabic (Kasim, 2014b), the highest /q/ VOT mean value obtained was $33 \mathrm{~ms}$. initially in a stressed syllable. It is suggested in this study that these short VOT values do not contribute in capturing intricate contextual variability as the one being investigated here.

Although it was tempting to hypothesize that /q/ VOT values could be greater in words containing final / $t$ / than those containing final /d/ (which would have accounted for vowel durations being shorter before / $t$ / and longer before $/ \mathrm{d} /$ ), the comparison did not reveal any significant difference. Speech variability in general and VOT variability in particular are very difficult to capture. Peterson and Lehiste (1960), for example, did not find any effect between vowel durations and prevocalic environment. In addition, Van Santen (1992) found that in utterance final position (similar to the context being examined in the present study) "the effects of pre-vocalic voicing were ...only $6 \mathrm{~ms}^{\prime \prime}$ (p. 531), which indicates that this context may reveal minimum influence, if any, on vowel duration.

As for vowel quality, it has been shown that different vowel qualities contribute to the discriminability of words with final voiceless and voiced stops. In a study that investigated the three vowels /I/, /i/, and /a/
(Lucy \& Charles-Lucy, 1985), it was found that "the difference between vowels preceding voiced and voiceless stops was greatest for test words containing /i/..., least for test words containing /I/.., and intermediate for test words containing /a/" (p. 1952). The occurrence of the three Arabic long vowels /a:/, /u:/ and /i:/ in the test data of the present study did not have any effect on the VOT values of the initial /q/.

\section{Conclusion}

In this study an attempt was made to explain vowel duration variation in syllables ending with voiceless and voiced stops in Arabic by exploring the VOT of an initial stop, viz. /q/. The three Arabic long vowels /a:/, /u:/ and /i:/ were used in a CVC context preceded by /q/ and followed by /t/ and /d/. The idea was to find out whether a correlation between the VOT of the initial stop and vowel durational differences could be established. However, the comparison between the initial stop VOT values in words ending with / $\mathrm{t} /$ and those ending with /d/ did not yield any significant difference. Although the three Arabic long vowels /a:/, /u:/ and /i:/ tend to show durational differences before final $/ \mathrm{t} /$ and $/ \mathrm{d} /$, they do not seem to be affected by prevocalic context. It appears that the search for a satisfactory explanation remains to be the goal of further research.

Appendix: VOT values of the initial stop / $\mathrm{q} /$ preceding the three Arabic long vowels /a:/, /u:/ and /i:/ (in $\mathrm{ms}$.). Note: $\mathrm{s}$ indicates subject, $\mathrm{t}$ indicates token.

\begin{tabular}{|c|c|c|c|c|c|c|c|}
\hline \multicolumn{2}{|c|}{} & qa:t & qa:d & qu:t & qu:d & qi:t & qi:d \\
\hline \multirow{3}{*}{ s1 } & t1 & 33 & 28 & 47 & 50 & 22 & 27 \\
\cline { 2 - 8 } & t2 & 25 & 34 & 30 & 46 & 19 & 45 \\
\cline { 2 - 8 } & t3 & 26 & 20 & 42 & 40 & 22 & 20 \\
\hline \multirow{3}{*}{ s2 } & t1 & 38 & 21 & 24 & 25 & 16 & 38 \\
\cline { 2 - 8 } & t2 & 27 & 28 & 29 & 25 & 28 & 17 \\
\cline { 2 - 8 } & t3 & 25 & 28 & 31 & 14 & 16 & 20 \\
\hline
\end{tabular}




\begin{tabular}{|c|c|c|c|c|c|c|c|}
\hline \multirow{3}{*}{ s3 } & $\mathrm{t} 1$ & 46 & 28 & 22 & 29 & 21 & 30 \\
\hline & t2 & 20 & 41 & 29 & 27 & 13 & 18 \\
\hline & t3 & 28 & 32 & 29 & 29 & 20 & 21 \\
\hline & $\mathrm{t} 1$ & 16 & 19 & 16 & 18 & 28 & 20 \\
\hline & t2 & 25 & 26 & 17 & 25 & 17 & 24 \\
\hline & $\mathrm{t} 3$ & 24 & 17 & 17 & 19 & 15 & 28 \\
\hline \multirow{3}{*}{ s5 } & $\mathrm{t} 1$ & 28 & 20 & 49 & 42 & 23 & 43 \\
\hline & t2 & 28 & 51 & 38 & 28 & 39 & 49 \\
\hline & $\mathrm{t} 3$ & 28 & 28 & 29 & 30 & 28 & 36 \\
\hline \multirow{3}{*}{ s6 } & $\mathrm{t} 1$ & 54 & 55 & 40 & 38 & 55 & 28 \\
\hline & $\mathrm{t} 2$ & 27 & 26 & 55 & 49 & 28 & 28 \\
\hline & $\mathrm{t} 3$ & 35 & 35 & 38 & 29 & 60 & 54 \\
\hline \multirow{3}{*}{ s7 } & $\mathrm{t} 1$ & 17 & 20 & 16 & 16 & 17 & 17 \\
\hline & $\mathrm{t} 2$ & 23 & 26 & 21 & 23 & 25 & 16 \\
\hline & $\mathrm{t} 3$ & 28 & 38 & 14 & 26 & 23 & 22 \\
\hline \multirow{3}{*}{ s8 } & $\mathrm{t} 1$ & 29 & 17 & 26 & 24 & 37 & 27 \\
\hline & t2 & 21 & 21 & 28 & 16 & 40 & 24 \\
\hline & t3 & 15 & 18 & 25 & 16 & 23 & 24 \\
\hline \multirow{3}{*}{ s9 } & $\mathrm{t} 1$ & 25 & 12 & 27 & 29 & 21 & 17 \\
\hline & $\mathrm{t} 2$ & 25 & 37 & 25 & 29 & 26 & 18 \\
\hline & $\mathrm{t} 3$ & 26 & 25 & 29 & 29 & 20 & 24 \\
\hline \multirow{3}{*}{$\begin{array}{l}\text { s1 } \\
0\end{array}$} & $\mathrm{t} 1$ & 35 & 28 & 29 & 43 & 48 & 26 \\
\hline & t2 & 38 & 33 & 29 & 29 & 40 & 42 \\
\hline & $\mathrm{t} 3$ & 30 & 30 & 21 & 29 & 41 & 48 \\
\hline
\end{tabular}

spectrographic study. Manuscript submitted for publication, Journal of Education and Science.

5. Ladefoged, P., \& Maddieson, I. (1996). The Sounds of the World's Languages. Oxford: Blackwell.

6. Lucy, P. A., \& Charles-Lucy, J. (1985). Contextual effects on vowel duration, closure duration, and the consonant/vowel ratio in speech production. J. Acoust. Soc. Am., 76, 1949-1957.

7. Maddieson, I. (1997). Phonetic universals. In: W. J. Hardcastle, \& J. Laver (Eds.), The Handbook of Phonetic Sciences (pp. 619-639). Oxford: Blackwell.

8. Metz, D. E., Allen, K., Kling, T., Maisonet, S., McCullough, R., Schiavetti, N., \& Whitehead, R. L. (2006). Effect of syllable-initial voicing on vowel duration during simultaneous communication. Journal of Communication Disorders, 39, 192-199.

9. Naeser, M. A. (1970). Influence of initial and final consonants on vowel duration in CVC syllables. Technical Report NO. 130. Wisconsin Research and Development, Center for Cognitive Learning, The University of Wisconsinc/Madison, Wisconsin.

10. Peterson, G. E., \& Lehiste, I. (1960). Duration of syllable nuclei in English, J. Acoust. Soc. Am., 62, 693-703.

11. Port, R. F., \& Dalby, J. (1982). Consonant/vowel ratio as a cue for voicing in English. Perception \& Psychophysics, 32, 141-152.

12. Reetz, H., \& Jongman, A. (2009). Phonetics: transcription, production, acoustics and perception. West Sussex: John Wiley and Sons.

\section{References}

1. Allen, J. S., \& Miller, J. L. (1999). Effects of syllable-initial voicing and speaking rate on the temporal characteristics of monosyllabic words. J. Acoust. Soc. Am., 106, 2031-9.

2. Cho, T., \& Ladefoged, P. (1999). Variation and universals in VOT: evidence from 18 languages. Journal of Phonetics, 27, 207-227.

3. Kasim, Ziyad R. (2014a). The effect of voiceless-voiced consonants on vowel duration in Arabic. Manuscript submitted for publication, Journal of Education and Science.

4. Kasim, Ziyad R. (2014b). Aspiration in Arabic: a 